\title{
Variation in term birthweight across European countries affects the prevalence of small for gestational age among very preterm infants
}

Jennifer Zeitlin (Jennifer.zeitlin@inserm.fr) ${ }^{1}$, Anna-Karin Edstedt Bonamy ${ }^{2}$, Aurelie Piedvache ${ }^{1}$, Marina Cuttini ${ }^{3}$, Henrique Barros ${ }^{4,5}$, Patrick Van Reempts ${ }^{6}$, Jan Mazela ${ }^{7}$, Pierre-Henri Jarreau ${ }^{8}$, Ludwig Gortner ${ }^{9}$, Elizabeth S. Draper ${ }^{10}$, Rolf F. Maier ${ }^{11}$, the EPICE Research Group ${ }^{\dagger}$

1.INSERM, Obstetrical, Perinatal and Pediatric Epidemiology Research Team, Centre for Epidemiology and Biostatistics (U1 153), Paris-Descartes University, Paris, France 2.Clinical Epidemiology Unit, Department of Medicine Solna and Department of Women's and Children's Health, Karolinska Institutet, Stockholm, Sweden

3.Clinical Care and Management Innovation Research Area, Bambino Gesù Children's Hospital, IRCCS, Rome, Italy

4.EPIUnit-Institute of Public Health, University of Porto, Porto, Portugal

5.Department of Clinical Epidemiology, Predictive Medicine and Public Health, University of Porto Medical School, Porto, Portugal

6.Department of Neonatology, Antwerp University Hospital, University of Antwerp, Edegem and Study Centre for Perinatal Epidemiology Flanders, Brussel, Belgium

7.Poznan University of Medical Sciences, Poznan, Poland

8.Université Paris V René Descartes and Assistance Publique Hôpitaux de Paris, Hôpitaux Universitaire Paris Centre Site Cochin, Service de Médecine et Réanimation néonatales de Port-Royal, Paris, France

9.Children's Hospital, University Hospital, University of Saarland, Homburg/Saar, Germany

10.Department of Health Sciences, University of Leicester, Leicester, UK

11.Children's Hospital, University Hospital, Philipps University, Marburg, Germany

\section{Keywords}

Birthweight, Growth restriction, Intrauterine growth references, Small for gestational age, Very preterm infants

\section{Correspondence \\ Jennifer Zeitlin, Obstetrical, Perinatal and Pediatric Epidemiology Research Team, Centre for \\ Epidemiology and Biostatistics, INSERM U1 153, 53 avenue de l'Observatoire, 75014 Paris, France. \\ Tel: 33142345577 | \\ Fax: 33143268979 \\ Email: Jennifer.zeitlin@inserm.fr}

\section{Received}

17 December 2016; revised 18 February 2017; accepted 3 April 2017.

DOI:10.1111/apa.13899

†EPICE Research Group members are listed in Appendix.

\begin{abstract}
Aim: This study assessed the prevalence of small for gestational age (SGA) among very preterm (VPT) infants using national and European intrauterine references.

Methods: We generated country-specific and common European intrauterine growth references for 11 European countries, according to Gardosi's approach and Hadlock's foetal growth model, using national data on birthweights by sex. These references were applied to the Effective Perinatal Intensive Care in Europe (EPICE) cohort, which comprised 7766 live VPT births without severe congenital anomalies under 32 weeks of gestation in $2011-2012$, to estimate the prevalence of infants with SGA birthweights, namely those below the 10th percentile.
\end{abstract}

Results: The SGA prevalence was 31.8\% with country-specific references and 34.0\% with common European references. The European references yielded a 10-point difference in the SGA prevalence between countries with lower term birthweights (39.9\%) - Portugal, Italy and France - and higher term birthweights, namely Denmark, the Netherlands, Sweden (28.9\%; $\mathrm{p}<0.001)$. This was not observed with country-specific references, where the respective figures were $32.4 \%$ and $33.9 \%(p=0.34)$, respectively.

Conclusion: One-third of VPT infants were SGA according to intrauterine references. Common European references showed significant differences in SGA prevalence between countries with high and low-term birthweights.

\section{INTRODUCTION}

Neonatal growth references based on birthweight are not appropriate for very preterm (VPT) infants, who are more likely to have abnormal foetal growth than infants born at term. Intra-uterine growth references make it possible to avoid this bias, as they are based on estimated foetal weights calculated from ultrasound measures in normal pregnancies ending at term. Between $25 \%$ and $35 \%$ of VPT infants are

\footnotetext{
Abbreviations

CA, Congenital anomalies; CV, Coefficient of variation; EPICE, Effective Perinatal Intensive Care in Europe; FGR, Foetal growth restriction; GA, Gestational age; HELLP, Haemolysis, elevated liver enzymes, low platelet count; SGA, Small for gestational age; VPT, Very preterm.
}

classified as small for gestational age (SGA) when intrauterine curves are used, as they have a birthweight below

\section{Key notes}

- We assessed the prevalence of small for gestational age (SGA) among 7766 very preterm infants from 11 countries in the Effective Perinatal Intensive Care in Europe cohort.

- We compared European intra-uterine growth references with national references that adjusted for term birthweight in each country.

- When common European references were used, SGA was more prevalent in countries with low-term birthweight than in countries with high-term birthweight. 
the 10th percentile (1-4). Moreover, using intra-uterine curves instead of neonatal growth curves improves predictions of infant mortality and morbidity risks $(5,6)$.

Nonetheless, many studies of SGA among VPT infants continue to use birthweight references $(7,8)$. This is probably explained by the availability of national birthweight references in most countries, whereas, with some exceptions $(4,9)$, national intra-uterine references are lacking. One approach to generating national intra-uterine references has been to adapt methods designed to customise individual growth references $(10,11)$. This method, proposed by Gardosi in the 1990s, models foetal growth using Hadlock's intra-uterine references (12), and adjusts them for individual physiological factors, such as maternal parity, height, weight and ethnicity (13). Population-level parameters may be used as well $(10,11)$. This methodology has been used to compare SGA across countries (11), but it has not been specifically applied to VPT infants.

Whether the population parameters should be national or international is also a matter of debate. The Intergrowth 21st Project, which collected foetal parameters in samples of healthy pregnancies in eight countries to develop one common set of references (14), is based on the hypothesis that, when mothers' nutritional and health needs are met, foetal growth is similar across geographical and ethnic settings (15). However, this hypothesis has not been tested, and it is not known whether country-level differences in birthweight affect percentiles for VPT infants.

This study aimed to describe the prevalence of SGA among VPT infants using Gardosi's methodology, overall and for key subgroups, and to evaluate the impact of using country-specific versus common European references.

\section{METHODS}

\section{Data}

This analysis used data from two European projects: EuroPeristat and Effective Perinatal Intensive Care in Europe (EPICE).

The Euro-Peristat project aims to monitor perinatal health in European countries using standardised indicators computed from routinely collected population-based data. It has produced two reports on the health and care of pregnant women and babies in Europe based on 2004 and 2010 data (16). In 2010, 29 countries participated in EuroPeristat. Data on average birthweights by sex, and standard deviations, were collected for live births at 40 weeks of gestation. Gestational age (GA) was defined as the best obstetric estimate using the last menstrual period and ultrasound measures, and the data were requested in completed weeks. Most European countries used firsttrimester ultrasounds for pregnancy dating in 2010.

The EPICE project aims to assess the use of evidencebased medicine for the care of very preterm infants (17). In 2011-2012, a geographically defined cohort of VPT stillbirths and live births from $22+0$ weeks to $31+6$ weeks of gestation was established in 19 European regions in 11 countries: Belgium (Flanders); Denmark (the Eastern
Region); Estonia (entire country); France (Burgundy, Ile-deFrance and the Northern regions), Germany (Hesse and Saarland); Italy (Emilia-Romagna, Lazio and Marche regions); the Netherlands (Central and Eastern regions); Poland (Wielkopolska); Portugal (Lisbon and Northern regions); Sweden (Stockholm County) and the UK (East Midlands, Northern and Yorkshire and Humber regions). Data were collected prospectively on births between April 2011 and September 2012 over a 12-month period in 10 countries and six months in France. More than 850000 births took place in the EPICE Regions in 2011. GA was defined based on the best obstetric assessment, using information on the last menstrual period and ultrasound measures, and collected as weeks and days. When there were several estimates, the following hierarchy was used to determine GA for the study: in vitro fertilisation treatment, ultrasound based on the earliest estimate, the last menstrual period, fundal height measurement and neonatal assessment at birth. All participating regions used first-trimester ultrasounds for pregnancies in 2011-2012.

\section{Study population}

The EPICE cohort comprised 10329 total births, of which 7900 were live births. Our inclusion criteria for this study were a live birth without a severe congenital anomaly (CA). We excluded stillbirths, because birth can occur several days or more after foetal death, complicating the assessment of their birthweight for gestational age. Infants with severe CA were excluded $(n=126,1.6 \%)$ because CA can have an impact on foetal weight. Babies with missing birthweight $(n=2)$ and missing or indeterminate sex $(n=6)$ were also excluded. The final study sample comprised 7766 infants.

\section{Analysis strategy}

To develop intra-uterine growth references charts, we adapted the approach proposed by Gardosi, which is based on Hadlock's foetal growth equation (log (expected foetal weight $\left.)=0.578+0.332 * \mathrm{GA}-0.00354 * \mathrm{GA}^{2}\right)$. Gardosi models the growth trajectory generated by this formula so that it can be applied to any expected birthweight at 40 weeks of gestation and used to derive the expected birthweight at each week of gestation. In customised models, expected birthweight takes into account individual parameters for each infant, namely the mother's height, weight, parity and ethnicity. However, the model can also be applied to an expected birthweight in a population of births $(10,11)$. This expected, or mean birthweight at term, can be interpreted as the 50th percentile, because the distribution of birthweight is assumed to be normal. The coefficient of variation - the standard deviation divided by the mean - based on the standard deviation of birthweight observed in the population at term is used to compute the other percentiles.

Using this methodology and the Euro-Peristat data on mean birthweights at 40 completed weeks of gestation in European countries, we created separate country-specific references for boys and girls for the 11 countries in the EPICE project. We also created a common European 
reference based on the median value for average birthweight at 40 weeks in the 11 countries. We used a coefficient of variation corresponding to the median value in participating countries for all the models (12\%). Appendix S1 provides a detailed explanation and the equations for deriving the common and country-specific references.

We first compared the 3rd, 10th, 50th, 90th and 97th percentiles across the countries and with the common European model. We then applied our references to the EPICE population of VPT infants and calculated the proportions of SGA infants according to both the country-specific and the common references. We defined SGA as a birthweight $<10$ th percentile, severe SGA as $<3$ rd percentile and moderate SGA as $3 \mathrm{rd}-<10$ th percentile. All other infants, with birthweights $\geq 10$ th percentile, were classified as not SGA.

We computed rates of SGA for the entire cohort, and by multiplicity: singletons, twins and higher order multiples. Because growth restriction is itself a cause of medically indicated very preterm delivery, we also computed rates of SGA for a subgroup of infants whose foetal growth restriction (FGR) was unlikely to be the direct cause of their preterm birth. We defined this group as infants with a spontaneous onset of delivery and without an antenatal diagnosis of FGR or pre-eclampsia, eclampsia or haemolysis, elevated liver enzymes, low platelet count (HELLP) syndrome. Diagnosis of FGR was mentioned in the medical records as a suspicion of foetal growth restriction. Preeclampsia was defined as proteinuria and systolic blood pressure $\geq 140 \mathrm{mmHg}$ and, or, diastolic blood pressure $\geq 90 \mathrm{mmHg}$ occurring after gestational week $20+0$ in a woman who was normotensive before she became pregnant. Proteinuria was defined as $\geq 300 \mathrm{mg} / \mathrm{L}$ protein in a random specimen or an excretion of $300 \mathrm{mg}$ over 24 hours. Hypertension had to be confirmed by two separate measurements. HELLP syndrome was defined as a cluster of laboratory abnormalities including serum lactic dehydrogenase $>600 \mathrm{U} / \mathrm{L}$, serum aspartate aminotransferase or serum alanine aminotransferase $>70 \mathrm{U} / \mathrm{L}$ and a platelet count of $<100000 / \mathrm{mm}^{3}$. Eclampsia was defined as the onset of seizures in a woman with pre-eclampsia.

We then assessed the impact of using country-specific versus common references by computing the proportion of infants whose SGA status changed between these two references. We also carried out these comparisons for three groups of countries defined by their birthweight at term high, medium and low - which were identified by dividing the difference between the maximum and minimum country-level birthweights into three intervals.

In a final step, we assessed the concordance of our model with Marsal's intra-uterine references (4) in the EPICE Swedish region. These references were based on 798 ultrasound measurements from 86 low-risk pregnancies, which showed that the 50th percentile for birthweight at 40 weeks of gestation was $3717 \mathrm{~g}$ for boys and $3595 \mathrm{~g}$ for girls. These were almost identical to the Euro-Peristat mean birthweights at 40 weeks of GA in Sweden. We compared the $3 \mathrm{rd}, 10 \mathrm{th}, 50 \mathrm{th}$ and 90 th percentiles of the published intra-uterine curves with the Swedish references generated by our models and then compared the rates of SGA generated from these references among Swedish infants in the EPICE cohort (18).

\section{Statistical analysis}

We used chi-square tests to compare rates of SGA by country group, country and multiplicity. The association of SGA rates with term birthweight was assessed using logistic regression, with the country group as an ordinal variable, and also by representing each country by its mean term birthweight. We computed Kappa statistics to measure the degree of concordance between the two methods of SGA classifications: overall and by country. The analyses were carried out using Stata 13.0 SE (Stata Corporation, College Station, TX, USA).

\section{RESULTS}

Table 1 provides the mean birthweights and standard deviations at 40 weeks of gestation for girls and boys, as obtained by Euro-Peristat, in the 11 countries included in the EPICE cohort. Countries are sorted by the mean birthweight at 40 weeks for girls and boys. For boys, these ranged from $3440 \mathrm{~g}$ in Portugal and $3478 \mathrm{~g}$ in Italy to $3717 \mathrm{~g}$ in Sweden and $3751 \mathrm{~g}$ in Estonia. For girls, the range was from $3300 \mathrm{~g}$ in Portugal and $3332 \mathrm{~g}$ in Italy to $3598 \mathrm{~g}$ in Sweden and $3587 \mathrm{~g}$ in Estonia. The median for the 11 countries was $3611 \mathrm{~g}$ for boys and $3460 \mathrm{~g}$ for girls. Most standard deviations fell between the range of 410 and $450 \mathrm{~g}$ for boys and 390 and $430 \mathrm{~g}$ for girls, yielding coefficients of variation close to $12 \%$. Countries were

\begin{tabular}{|c|c|c|c|c|c|c|c|}
\hline \multirow[b]{3}{*}{ Country* } & \multirow[b]{3}{*}{$\mathrm{N}^{\dagger}$} & \multicolumn{3}{|l|}{ Males } & \multicolumn{3}{|c|}{ Females } \\
\hline & & \multicolumn{3}{|c|}{ Birthweight at 40 weeks } & \multicolumn{3}{|c|}{ Birthweight at 40 weeks } \\
\hline & & Mean & SD & CV (\%) & Mean & SD & CV $(\%)$ \\
\hline Portugal & 21305 & 3440 & 454 & 13.2 & 3300 & 455 & 13.8 \\
\hline Italy & 130671 & 3478 & 417 & 12.0 & 3332 & 399 & 12.0 \\
\hline France ${ }^{\ddagger}$ & 3999 & 3512 & 412 & 11.7 & 3379 & 394 & 11.7 \\
\hline UK: England & 195573 & 3587 & 443 & 12.4 & 3447 & 426 & 12.4 \\
\hline Poland & 131573 & 3603 & 444 & 12.3 & 3440 & 424 & 12.3 \\
\hline $\begin{array}{l}\text { Belgium } \\
\text { (Flanders) }\end{array}$ & 19782 & 3611 & 425 & 11.8 & 3460 & 401 & 11.6 \\
\hline Germany & 177781 & 3624 & 428 & 11.8 & 3475 & 415 & 11.9 \\
\hline Denmark & 17555 & 3697 & 443 & 12.0 & 3565 & 429 & 12.0 \\
\hline The Netherlands & 47591 & 3697 & 450 & 12.2 & 3552 & 433 & 12.2 \\
\hline Sweden & 31683 & 3717 & 447 & 12.0 & 3598 & 436 & 12.1 \\
\hline Estonia & 4962 & 3751 & 432 & 11.5 & 3587 & 421 & 11.7 \\
\hline Median value & - & 3611 & 443 & 12.0 & 3460 & 424 & 12.0 \\
\hline
\end{tabular}

*Countries sorted by the average of male and female mean birthweight values as data in Euro-Peristat were provided separately for males and females.

${ }^{\dagger}$ Number of singleton births at 40 weeks of GA.

${ }^{\ddagger}$ From a representative national sample of births. 
divided into low birthweight for Portugal, Italy and France, medium birthweights for England, Poland, Belgium and Germany and high birthweights for Denmark, the Netherlands, Sweden and Estonia).

The mean gestational age in our sample was 28.4 weeks, and the mean birthweight was $1192 \mathrm{~g}$ (Table 2). More than $30 \%$ of the infants were from multifoetal pregnancies. Antenatal detection of foetal growth restriction and hypertensive diseases of pregnancy were common $(16.2 \%$ and $14.7 \%$ respectively) and $40.6 \%$ of the deliveries resulted from a prelabour Caesarean. Infants at lower risk of FGR constituted $56.5 \%$ of the sample and this was defined as the spontaneous onset of delivery without suspicion of FGR or preeclampsia, eclampsia or HELLP.

Figure 1 illustrates the growth trajectory and shows the 3rd, 10th, 50th, 90th and 97th percentiles for girls and boys using values generated by the common European model. Table S1 of Appendix S1 provides values for all the countryspecific and common percentiles for each completed week of GA by sex. The country-specific percentiles were different between countries with low and high-term birthweights. The 10th percentile at 31 weeks, for instance, was 110 $130 \mathrm{~g}$ higher in the highest versus lowest birthweight countries. This means that the 10th percentile values in Portugal and in Italy were close to the 3rd percentile values in Estonia and Sweden across all the gestational ages considered in this study.

Table 3 describes rates of SGA for the EPICE cohort. Based on the country-specific references, $31.8 \%$ of the infants were SGA, with $20.5 \%$ under the 3rd percentile and $11.4 \%$ between the $3 \mathrm{rd}$ and 10 th percentiles. While rates of

\begin{tabular}{|c|c|c|}
\hline & \multicolumn{2}{|c|}{ Live births $(\mathrm{n}=7766)$} \\
\hline & Mean & SD \\
\hline Gestational age (weeks) & 28.4 & 2.4 \\
\hline \multirow{2}{*}{ Birthweight (g) } & 1192 & 401 \\
\hline & $\mathrm{N}$ & $\%$ \\
\hline Male & 4204 & 54.1 \\
\hline \multicolumn{3}{|l|}{ Type of gestation } \\
\hline Singleton & 5344 & 68.8 \\
\hline Multiple & 2144 & 27.6 \\
\hline Triplet/quadruplet & 276 & 3.6 \\
\hline Antenatal suspicion of FGR & 1233 & 16.2 \\
\hline Pre-eclampsia/Eclampsia/HELLP & 1145 & 14.7 \\
\hline \multicolumn{3}{|l|}{ Mode of delivery } \\
\hline Prelabour Caesarean & 3114 & 40.6 \\
\hline Intrapartum Caesarean & 1883 & 24.5 \\
\hline Vaginal delivery & 2683 & 34.9 \\
\hline $\begin{array}{l}\text { Spontaneous onset of delivery } \\
\text { with no antenatal suspicion of } \\
\text { FGR or pre-eclampsia/eclampsia/HELLP }\end{array}$ & 4344 & 56.5 \\
\hline
\end{tabular}

Variables do not sum up to total because of missing observations (2 for multiples, 6 for sex - also includes undetermined sex - and $<1 \%$ for other variables, $1.2 \%$ for composite variable on FGR).
SGA $<10$ th percentile were not significantly different for singletons and twins, more singleton SGA infants were severely SGA compared to twins, whereas twins and triplets were more likely to have a birthweight between the 3rd and 10th percentile. When pregnancy complications associated with FGR and nonspontaneous onset births were excluded, the rates of SGA declined to $14.8 \%$ of all births for the country-specific references and to $11.0 \%$ of singletons. In this population, twins were consistently more likely to be SGA. Overall, the rate of SGA $<10$ th percentile was higher between 28 and 31 weeks of GA $(1918 / 5294,36.2 \%)$ than before 28 weeks of GA $(579 / 2472,23.4 \% ; p=<0.001)$. Patterns of multiplicity, risk status and gestational age group were similar when using common European references, although the percentage of SGA was slightly higher $(34.0 \%$ vs $31.8 \%)$.

Table 4 presents the rates of SGA using country-specific and common references by individual country and country birthweight group. When the common references were used between $4.1 \%$ and $15.2 \%$ of infants were reclassified in high and low birthweight countries, leading to a 10 point difference in SGA prevalence between high versus low birthweight countries $(38.9 \%, 31.3 \%$ and $28.9 \%$, respectively). The $\mathrm{p}$ for trend was $<0.001$, and $\mathrm{p}$ value was $\mathrm{p}<0.001$ when we compared high and low birthweight countries. This was not the case for the country-specific references: the $\mathrm{p}$ for trend was 0.96 , the $\mathrm{p}$ value for the comparison between high to low birthweight countries was $\mathrm{p}=0.34$, and the $\mathrm{p}$ value for the comparison of the three groups was $\mathrm{p}=0.03$. For individual countries, there was a strong trend associating SGA rates with average birthweight at term when the common references were used $(\mathrm{p}<0.001)$, which was not significant using countryspecific references $(p=0.94)$. The Kappa statistics, which provided a measure of concordance between these classifications, were very high in most cases, but they were lower for the low and high birthweight country groups than for the medium group and in some individual countries, such as Portugal, Estonia and Sweden. Table 4 also illustrates the variability in rates of SGA by country in the regions participating in EPICE, even when country-specific references were used, from lows of $25.8 \%$ and $28.4 \%$ in Poland and Belgium to highs of 36.9\% and 37.5\% in Germany and Denmark, respectively.

Figure 2 compares the model used for this analysis, based on Hadlock's references and Gardosi approach, labelled the Hadlock-Gardosi Model, with the Swedish references proposed by Marsal et al. for girls and boys (4). The Marsal et al.'s curve was slightly higher than the Hadlock-Gardosi Model, especially for girls, and the differences were more marked for the 50th and 90th percentiles than for the 3rd and 10th. Overall 35.1\% of Swedish VPT infants were under the 10th percentile using Swedish national references versus $31.5 \%$ for the Hadlock-Gardosi model and $23.9 \%$ and $21.5 \%$, respectively were under the 3rd percentile (Table S2 of Appendix S1). The proportions were closer for boys (32.4\% vs $31.7 \%$ ) than girls (38.4\% vs $31.3 \%$ ). Concordance was $96.4 \%: 99.3 \%$ for boys and $92.1 \%$ for girls. 


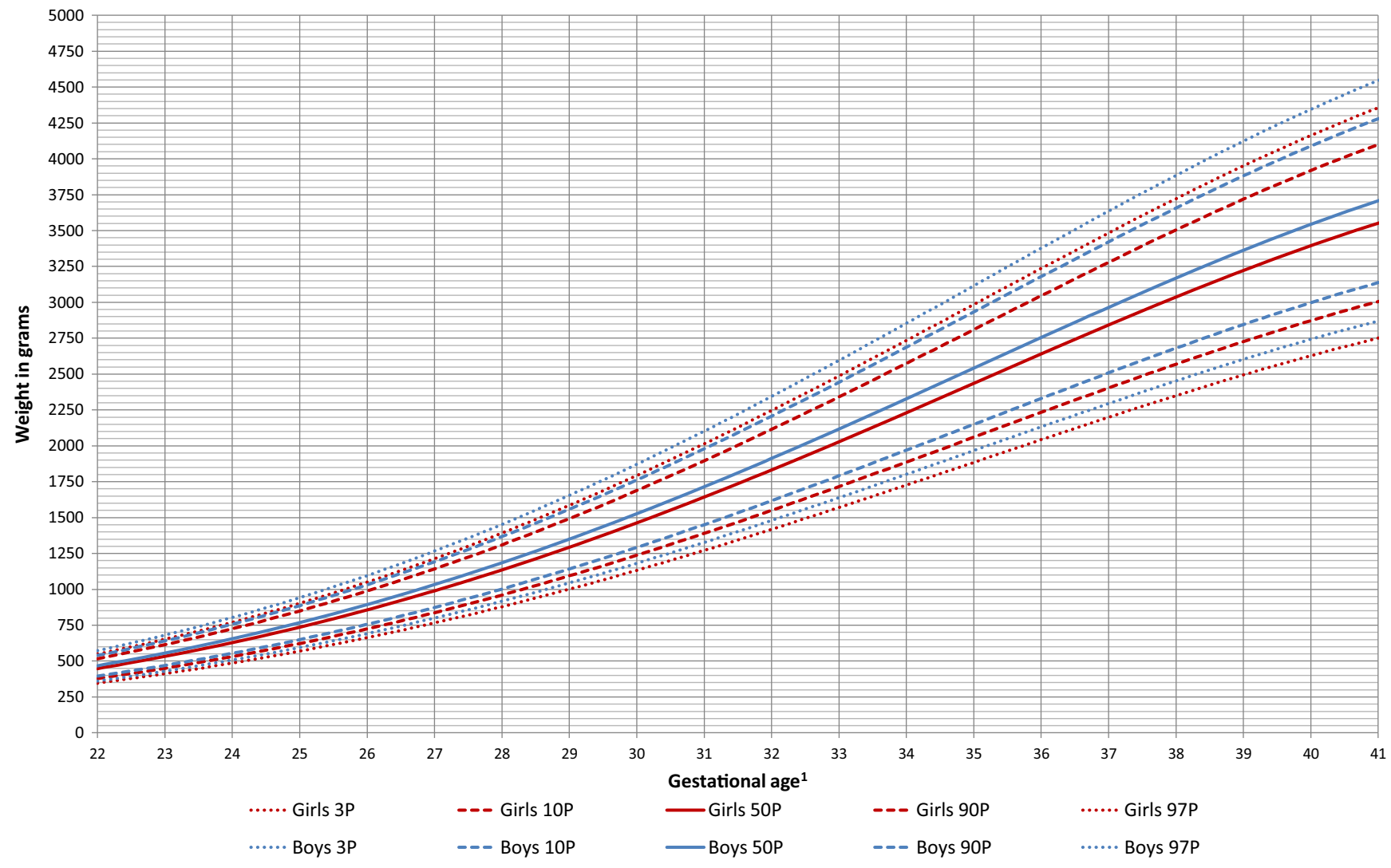

Figure 1 Percentile values for intra-uterine growth references for very preterm boys and girls using the common European model. Note: 1. Gestational age corresponds to exact weeks of gestation, that is $22=22+0$ days.

Table 3 Rates of SGA for all infants and infants at lower risk of FGR by multiplicity using country-specific and common European references

\begin{tabular}{|c|c|c|c|c|c|c|c|}
\hline & \multirow[b]{2}{*}{$\mathrm{n}$} & \multicolumn{3}{|c|}{ Country-specific references } & \multicolumn{3}{|c|}{ Common European references } \\
\hline & & $\begin{array}{l}<3 r d p \\
\%\end{array}$ & $\begin{array}{l}\text { 3rd-9th } p \\
\%\end{array}$ & $\begin{array}{l}\text { Total }<10 \text { th } \mathrm{p} \\
\%\end{array}$ & $\begin{array}{l}<3 r d \mathrm{P} \\
\%\end{array}$ & $\begin{array}{l}\text { 3rd-9th } \mathrm{p} \\
\%\end{array}$ & $\begin{array}{l}\text { Total }<10 \text { th } \mathrm{p} \\
\%\end{array}$ \\
\hline Overall by multiplicity & 7766 & 20.5 & 11.4 & 31.8 & 21.8 & 12.2 & 34.0 \\
\hline Singleton & 5344 & 22.1 & 10.0 & 32.0 & 23.4 & 10.3 & 33.8 \\
\hline Twin & 2144 & 16.3 & 14.3 & 30.6 & 17.5 & 15.9 & 33.4 \\
\hline Triplet/quadruplet & 276 & 21.7 & 15.6 & 37.3 & 23.2 & 18.5 & 41.7 \\
\hline P value (twins vs. singletons) & & $<0.01$ & $<0.001$ & 0.226 & $<0.001$ & $<0.001$ & 0.759 \\
\hline P value (triplets vs. singletons) & & 0.670 & 0.002 & 0.067 & 0.610 & $<0.001$ & 0.012 \\
\hline Lower risk of FGR* by multiplicity & 4344 & 5.7 & 9.1 & 14.8 & 6.3 & 10.6 & 16.9 \\
\hline Singleton & 2854 & 4.1 & 6.8 & 11.0 & 4.7 & 7.9 & 12.5 \\
\hline Multiple & 1347 & 8.3 & 13.4 & 21.8 & 9.2 & 15.5 & 24.7 \\
\hline Triplet/quadruplet & 141 & 10.6 & 14.2 & 24.8 & 10.6 & 19.1 & 29.8 \\
\hline P value (twins vs. singletons) & & $<0.010$ & $<0.001$ & $<0.001$ & $<0.001$ & $<0.001$ & $<0.001$ \\
\hline P value (triplets vs. singletons) & & $<0.001$ & $<0.001$ & $<0.001$ & $<0.001$ & $<0.001$ & $<00.001$ \\
\hline
\end{tabular}

\section{DISCUSSION}

We constructed country-specific and common European intra-uterine growth references for VPT infants using data on birthweight at 40 weeks of gestation in 11 European countries. Mean birthweight at term differed by more than $300 \mathrm{~g}$ across countries and this led to substantial differences in the 3rd and 10th percentiles for VPT infants in high versus low-term birthweight countries. Using countryspecific references, $32 \%$ of VPT infants in our European cohort were SGA and most had birthweights under the 3rd percentile. The use of a common versus country-specific reference classified a larger proportion of infants as SGA in 


\begin{tabular}{|c|c|c|c|c|c|c|c|c|c|}
\hline & \multirow[b]{2}{*}{$\mathrm{N}$} & \multicolumn{3}{|c|}{ Country-specific references } & \multicolumn{3}{|c|}{ Common European references } & \multirow[b]{2}{*}{$\begin{array}{l}\text { Change SGA status } \\
\%\end{array}$} & \multirow[b]{2}{*}{$\begin{array}{l}\text { Kappa } \\
\text { Statistic }\end{array}$} \\
\hline & & $\begin{array}{l}<3 r d \mathrm{P} \\
\%\end{array}$ & $\begin{array}{l}\text { 3rd-9th } p \\
\%\end{array}$ & $\begin{array}{l}\text { Total }<10 \text { th } p \\
\%\end{array}$ & $\begin{array}{l}<3 \text { rd P } \\
\%\end{array}$ & $\begin{array}{l}\text { 3rd-9th } \mathrm{p} \\
\%\end{array}$ & $\begin{array}{l}\text { Total }<10 \text { th } p \\
\%\end{array}$ & & \\
\hline Total & 7766 & 20.5 & 11.4 & 31.8 & 21.8 & 12.2 & 34.0 & 6.0 & 0.92 \\
\hline \multicolumn{10}{|l|}{ Individual countries* } \\
\hline Portugal & 719 & 21.4 & 11.7 & 33.1 & 26.8 & 15.6 & 42.4 & 14.7 & 0.74 \\
\hline Italy & 1111 & 19.4 & 11.7 & 31.1 & 24.3 & 13.6 & 37.9 & 11.8 & 0.81 \\
\hline France & 1290 & 22.2 & 10.9 & 33.2 & 24.8 & 12.9 & 37.8 & 7.1 & 0.85 \\
\hline UK & 1714 & 18.6 & 11.1 & 29.8 & 19.3 & 11.5 & 30.8 & 1.8 & 0.96 \\
\hline Poland & 299 & 13.0 & 12.7 & 25.8 & 13.4 & 13.7 & 27.1 & 1.7 & 0.91 \\
\hline Belgium & 749 & 17.4 & 11.1 & 28.4 & 17.4 & 11.9 & 29.2 & 0.8 & 0.99 \\
\hline Germany & 731 & 24.6 & 12.3 & 36.9 & 24.1 & 12.2 & 36.3 & 1.2 & 0.98 \\
\hline Denmark & 347 & 26.2 & 11.2 & 37.5 & 24.2 & 8.4 & 32.6 & 6.9 & 0.85 \\
\hline The Netherlands & 392 & 23.7 & 9.2 & 32.9 & 21.9 & 8.7 & 30.6 & 4.1 & 0.90 \\
\hline Sweden & 263 & 21.3 & 10.3 & 31.6 & 16.3 & 9.9 & 26.2 & 10.3 & 0.78 \\
\hline Estonia & 151 & 17.2 & 15.2 & 32.5 & 13.9 & 6.6 & 20.5 & 15.2 & 0.75 \\
\hline $\mathrm{P}$ value (chi-square) & & & & $<0.001$ & & & $<0.001$ & & \\
\hline p value (test for trend) & & & & 0.94 & & & $<0.001$ & & \\
\hline \multicolumn{10}{|l|}{ Country groups ${ }^{\dagger}$} \\
\hline Low-term birthweight countries & 3120 & 21.0 & 11.4 & 32.4 & 25.1 & 13.8 & 38.9 & 10.5 & 0.87 \\
\hline Medium-term birthweight countries & 3493 & 19.1 & 11.5 & 30.6 & 19.4 & 11.9 & 31.3 & 1.4 & 0.98 \\
\hline High-term birthweight countries & 1153 & 23.1 & 10.8 & 33.9 & 20.3 & 8.6 & 28.9 & 7.8 & 0.88 \\
\hline p value (chi-square) & & & & 0.03 & & & $<0.001$ & & \\
\hline p value (test for trend) & & & & 0.96 & & & $<0.001$ & & \\
\hline
\end{tabular}

*Countries sorted by average birthweight at 40 weeks of boys and girls.

†Low-term birthweight: Portugal, Italy, France; Medium-term birthweight: England, Poland, Belgium and Germany; High-term birthweight: Denmark, the Netherlands, Sweden and Estonia.

low birthweight countries and a lower proportion in high birthweight countries.

Our study found that about one-third of VPT infants had a birthweight that was less than the 10th percentile, and $20 \%$ were less than the $3 \mathrm{rd}$ percentile. This is consistent with other research on SGA rates when intrauterine references were used (1-4). In Sweden, Marsal et al. found that $32 \%$ of infants born before 30 weeks of gestation had a birth weight below the 3rd percentile, compared to $11 \%$ at $30-36$ weeks (4). Zaw et al., who studied infants born before 34 weeks of gestation, found that $12 \%$ had birthweights under the 10th percentile according to neonatal standards, but this rose to $23 \%$ when foetal growth standards were used (1). Ferdynus et al. showed that $25 \%$ of French births under 37 weeks were under the 10th percentile of foetal weight standards versus $11 \%$ by birth weight standards (19). In our sample, the prevalence of SGA was lower when gestational age was under 28 weeks, which may reflect fewer indicated deliveries for growth restriction among extremely preterm infants. The SGA prevalence also differed substantially by multiplicity and pregnancy complications, which is in line with current knowledge about the impact of these clinical parameters on growth restriction in the setting of prematurity (20).

We found a strong association between rates of SGA and mean term birthweight when a common reference was used. These results call into question the use of common growth norms, as advocated by the Intergrowth 21 st project (15). Basing evaluations of SGA on our common model would require the assumption that the large differences in term birthweight in Europe resulted primarily from abnormal growth as opposed to physiological differences in population size. Previous European research has provided support for this latter assertion, as the optimal birthweight defined as the birthweight at which mortality is lowest - is lower in populations with lower term birthweights (21). Other studies have shown that using population-adjusted references more adequately identifies infants at risk of poor outcome (22). The National Institute of Child Health and Development Fetal Growth Studies also contested this claim with its recently published references by race and ethnicity in the United States (23).

Even when we used country-specific references, we found heterogeneity in the SGA prevalence between EPICE regions. These differences could have resulted from population characteristics, such as maternal age or the multiple pregnancy rate, which vary in Europe and impact on the prevalence and causes of preterm birth (24). Different healthcare policies and practices, including prenatal care and screening, the criteria for inducing delivery because of foetal growth impairment and approaches to ethical decision-making may also play a role. The contribution of these factors to rates of SGA among very preterm infants is an important area for further research. The existence of this variability across countries provides further justification for 

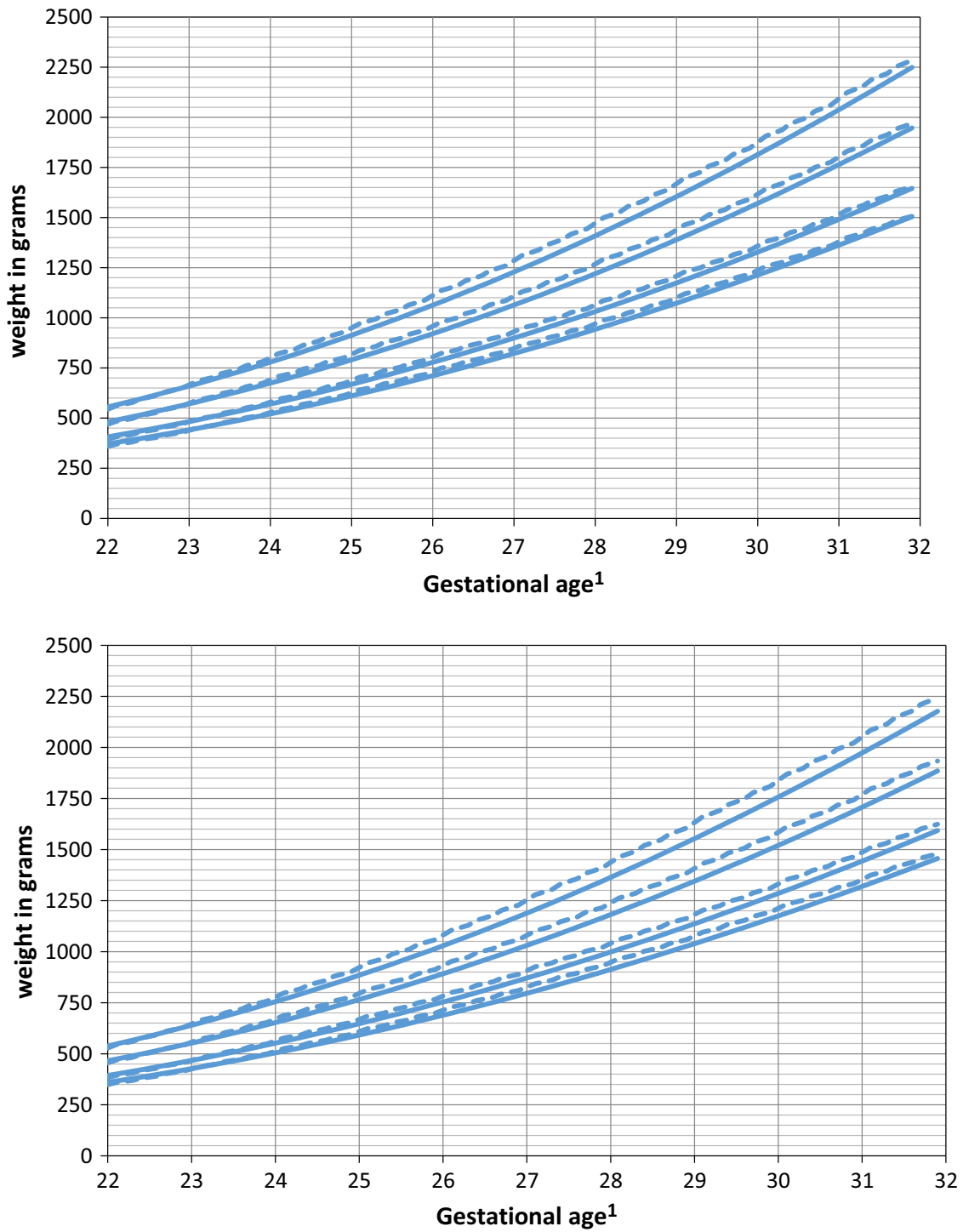

Figure 2 Comparison between the 3rd, 10th, 50th and 90th percentiles of Hadlock-Gardosi (HG) model (2a) and Marsal's Swedish intra-uterine references (2b) for males and females. (a) Males - Hadlock-Gardosi (solid line), Marsal (dotted line). (b) Females - Hadlock-Gardosi (solid line), Marsal (dotted line). Note: 1 corresponds to exact weeks of gestation, that is $22=22+0$ days.

avoiding neonatal birthweight standards, which, by definition, classify $10 \%$ of the population under the 10th percentile and therefore do not make it possible to assess or investigate these differences.

Our study had potential limitations. The first limitation was that we assumed that mean term birthweights could be used to create country-specific references, but this may not have been appropriate for all infants, especially in countries with large migrant populations. Taking into account maternal height, weight and ethnicity could make it possible to refine these references, but these data were not available. Furthermore, studies have shown that these maternal characteristics only account for a small proportion of the birthweight variability, especially for VPT infants (2). The second limitation was that our references relied on the untested assumption that Hadlock's formula could be transposed to any population and that the coefficient of variation remains constant across all gestational ages. Nevertheless, the Hadlock's growth references and Gardosi's customised model are widely used in multiple countries for clinical care and research (25) and many intrauterine growth models assume that the coefficient of variation is constant $(4,26,27)$. We were able to compare our model with Swedish national intra-uterine references for the Swedish infants in our sample and observed high concordance for SGA prevalence, despite slight differences in percentile values, which have been noted in other comparisons (9). These differences may result from the use of various statistical methods (28) and changes in foetal growth over time. The publication of intra-uterine curves by the Intergrowth 21st project (29) the World Health Organization study of foetal growth (30) and the 
National Institute of Child Health and Development foetal growth project (23) provides an opportunity for future comparative studies on the assumptions used in these models. Finally, future investigations should go beyond a statistical definition of SGA and include other clinical measures to assess growth restrictions, such as Doppler measurements, in order to verify the appropriateness of specific thresholds across populations using more specific measures of morbidity.

\section{CONCLUSION}

This study constructed country-specific intra-uterine growth references for 11 countries in Europe based on the Hadlock-Gardosi model and illustrated the high proportions of very preterm infants classified as SGA, and, in particular, as severely SGA with a birthweight under the 3rd percentile when intra-uterine references were used. We showed that the large variation in term birthweight across European countries affected birthweight references for VPT infants, calling into question the use of common references for the assessment of SGA in VPT infants in Europe. Our results also highlight the need for cross-national studies on the causes and consequences of foetal growth restriction in this high-risk population.

\section{FUNDING STATEMENTS}

The research leading to these results received funding from the European Union's Seventh Framework Programme (FP7/2007-2013 under grant agreement $n^{\circ} 259882$ ). The Euro-Peristat project received funding from the European Union in the framework of the Health Programme (grant numbers 20101301 and 664691). Additional funding for the EPICE project is acknowledge from the following regions: France (French Institute of Public Health Research/Institute of Public Health and its partners the French Health Ministry, the National Institute of Health and Medical Research, the National Institute of Cancer and the National Solidarity Fund for Autonomy; grant ANR-11-EQPX-0038 from the National Research Agency through the French Equipex Program of Investments in the Future; and the PremUp Foundation); Poland (2012-2015 allocation of funds for international projects from the Polish Ministry of Science and Higher Education); Sweden [Stockholm County Council (ALF-project and Clinical Research Appointment) and by the Department of Neonatal Medicine, Karolinska University Hospital], and by the Department of Neonatal Medicine, Karolinska University Hospital), AKEB supported by a grant from the Swedish Research Council for Health, Working Life and Welfare (grant no 2010-0643); UK (funding for The Neonatal Survey from Neonatal Networks for East Midlands and Yorkshire \& Humber regions).

\section{CONFLICTS OF INTEREST}

The authors have no conflicts of interest to disclose.

\section{References}

1. Zaw W, Gagnon R, da Silva O. The risks of adverse neonatal outcome among preterm small for gestational age infants according to neonatal versus fetal growth standards. Pediatrics 2003; 111: 1273-7.

2. Charkaluk ML, Marchand-Martin L, Ego A, Zeitlin J, Arnaud C, Burguet A, et al. The influence of fetal growth reference standards on assessment of cognitive and academic outcomes of very preterm children. J Pediatr 2012; 161: 1053-8.

3. Zeitlin J, Ancel PY, Saurel-Cubizolles MJ, Papiernik E. The relationship between intrauterine growth restriction and preterm delivery: an empirical approach using data from a European case-control study. BJOG 2000; 107: 750-8.

4. Marsal K, Persson PH, Larsen T, Lilja H, Selbing A, Sultan B. Intrauterine growth curves based on ultrasonically estimated foetal weights. Acta Paediatr 1996; 85: 843-8.

5. Da Fre M, Polo A, Di Lallo D, Piga S, Gagliardi L, Carnielli V, et al. Size at birth by gestational age and hospital mortality in very preterm infants: results of the area-based ACTION project. Early Hum Dev 2015; 91: 77-85.

6. Guellec I, Lapillonne A, Renolleau S, Charlaluk ML, Roze JC, Marret S, et al. Neurologic outcomes at school age in very preterm infants born with severe or mild growth restriction. Pediatrics 2011; 127: e883-91.

7. Eryigit-Madzwamuse S, Wolke D. Attention problems in relation to gestational age at birth and smallness for gestational age. Early Hum Dev 2015; 91: 131-8.

8. van Dommelen P, Verkerk PH, van Straaten HL, Dutch Neonatal Intensive Care Unit Neonatal Hearing Screening Working G. Hearing loss by week of gestation and birth weight in very preterm neonates. $J$ Pediatr 2015; 166: 840-3 e1.

9. Johnsen SL, Rasmussen S, Wilsgaard T, Sollien R, Kiserud T. Longitudinal reference ranges for estimated fetal weight. Acta Obstet Gynecol Scand 2006; 85: 286-97.

10. Ego A, Subtil D, Grange G, Thiebaugeorges O, Senat MV, Vayssiere C, et al. Customized versus population-based birth weight standards for identifying growth restricted infants: a French multicenter study. Am J Obstet Gynecol 2006; 194: 1042-9.

11. Mikolajczyk RT, Zhang J, Betran AP, Souza JP, Mori R, Gulmezoglu AM, et al. A global reference for fetal-weight and birthweight percentiles. Lancet 2011; 377: 1855-61.

12. Hadlock FP, Harrist RB, Martinez-Poyer J. In utero analysis of fetal growth: a sonographic weight standard. Radiology 1991; 181: 129-33.

13. Gardosi J, Mongelli M, Wilcox M, Chang A. An adjustable fetal weight standard. Ultrasound Obst Gyn 1995; 6: 168-74.

14. Papageorghiou AT, Ohuma EO, Altman DG, Todros T, Cheikh Ismail L, Lambert A, et al. International standards for fetal growth based on serial ultrasound measurements: the Fetal Growth Longitudinal Study of the INTERGROWTH-21st Project. Lancet 2014; 384: 869-79.

15. Villar J, Papageorghiou AT, Pang R, Ohuma EO, Cheikh Ismail L, Barros FC, et al. The likeness of fetal growth and newborn size across non-isolated populations in the INTERGROWTH-21st Project: the Fetal Growth Longitudinal Study and Newborn Cross-Sectional Study. Lancet Diabetes Endocrinol 2014; 2: 781-92.

16. Zeitlin J, Mohangoo A, Cuttini M, Alexander S, Barros H, Blondel B, et al. The European Perinatal Health Report: comparing the health and care of pregnant women and newborn babies in Europe. J Epidemiol Community Health 2009; 63: 681-2. 
17. Zeitlin J, Manktelow BN, Piedvache A, Cuttini M, Boyle E, van Heijst A, et al. Use of evidence based practices to improve survival without severe morbidity for very preterm infants: results from the EPICE population based cohort. BMJ 2016; 354: i2976.

18. Villar J, Cheikh Ismail L, Victora CG, Ohuma EO, Bertino E, Altman DG, et al. International standards for newborn weight, length, and head circumference by gestational age and sex: the Newborn Cross-Sectional Study of the INTERGROWTH-21st Project. Lancet 2014; 384: 857-68.

19. Ferdynus C, Quantin C, Abrahamowicz M, Burguet A, Sagot P, Gouyon JB. Comparison of the ability of alternative birthweight and fetal weight standards to identify preterm newborns at increased risk of perinatal death. BJOG 2013; 120 : 1456-64.

20. Papiernik E, Zeitlin J, Delmas D, Blondel B, Kunzel W, Cuttini $\mathrm{M}$, et al. Differences in outcome between twins and singletons born very preterm: results from a population-based European cohort. Hum Reprod 2010; 25: 1035-43.

21. Graafmans WC, Richardus JH, Borsboom GJ, Bakketeig L, Langhoff-Roos J, Bergsjo P, et al. Birth weight and perinatal mortality: a comparison of "optimal" birth weight in seven Western European countries. Epidemiology 2002; 13: 569-74.

22. Urquia ML, Berger H, Ray JG. Canadian Curves C. Risk of adverse outcomes among infants of immigrant women according to birth-weight curves tailored to maternal world region of origin. Can Med Assoc J 2015; 187: E32-40.

23. Buck Louis GM, Grewal J, Albert PS, Sciscione A, Wing DA, Grobman WA, et al. Racial/ethnic standards for fetal growth: the NICHD Fetal Growth Studies. Am J Obstet Gynecol 2015; 213: 449 e1-41.

24. Delnord M, Blondel B, Zeitlin J. What contributes to disparities in the preterm birth rate in European countries? Curr Opin Obst Gyn 2015; 27: 133-42.

25. Gibbons KS, Chang AM, Flenady VJ, Mahomed K, Gray PH, Gardener GJ, et al. A test of agreement of customised birthweight models. Paediatr Perinat Epidemiol 2013; 27: 131-7.

26. Larsen T, Petersen S, Greisen G, Larsen JF. Normal fetal growth evaluated by longitudinal ultrasound examinations. Early Hum Dev 1990; 24: 37-45.

27. Secher NJ, Hansen PK, Lenstrup C, Pedersen-Bjergaard L, Eriksen PS, Thomsen BL, et al. Birthweight-for-gestational age charts based on early ultrasound estimation of gestational age. Br J Obstet Gynaecol 1986; 93: 128-34.
28. Ioannou C, Talbot K, Ohuma E, Sarris I, Villar J, CondeAgudelo A, et al. Systematic review of methodology used in ultrasound studies aimed at creating charts of fetal size. BJOG 2012; 119: 1425-39.

29. Stirnemann J, Villar J, Salomon LJ, Ohuma E, Ruyan P, Altman DG, et al. International estimated fetal weight standards of the INTERGROWTH-21st Project. Ultrasound Obst Gyn 2016. https://doi.org/10.1002/uog.17347.

30. Kiserud T, Piaggio G, Carroli G, Widmer M, Carvalho J, Neerup Jensen L, et al. The World Health Organization Fetal Growth Charts: A multinational longitudinal study of ultrasound biometric measurements and estimated fetal weight. PLoS Med 2017; 14: e1002220.

\section{APPENDIX}

The EPICE Research Group: BELGIUM Flanders (E Martens, G Martens, P Van Reempts); DENMARK Eastern Region (K Boerch, A Hasselager, L Huusom, O Pryds, T Weber); ESTONIA (L Toome, H Varendi); FRANCE Burgundy, Ile-de France and Northern Region (PY Ancel, B Blondel, A Burguet, PH Jarreau, P Truffert); GERMANY Hesse (RF Maier, B Misselwitz, S Schmidt), Saarland (L Gortner); ITALY Emilia Romagna (D Baronciani, G Gargano), Lazio (R Agostino, D DiLallo, F Franco), Marche (V Carnielli), M Cuttini; NETHERLANDS Eastern \& Central (C Koopman-Esseboom, A Van Heijst, J Nijman); POLAND Wielkopolska (J Gadzinowski, J Mazela); PORTUGAL Lisbon and Tagus Valley (LM Graça, MC Machado), Northern region (MRG Carrapato, T Rodrigues), H Barros; SWEDEN Stockholm (AK Bonamy, M Norman, E Wilson); UK East Midlands and Yorkshire and Humber (E Boyle, ES Draper, BN Manktelow), Northern Region (AC Fenton, DWA Milligan); INSERM, Paris (J Zeitlin, M Bonet, A Piedvache).

\section{SUPPORTING INFORMATION}

Additional Supporting Information may be found in the online version of this article:

Appendix S1 Modeling intrauterine growth references. 